\title{
Boron Neutron Capture Therapy (BNCT) - Low-Energy Neutron Spectrometer for Neutron Field Characterization -*)
}

\author{
Isao MURATA and Tsubasa OBATA \\ Division of Electrical, Electronic and Information Engineering, Graduate School of Engineering, Osaka University, \\ 2-1 Yamada-oka, Suita, Osaka 565-0871, Japan
}

(Received 24 July 2013 / Accepted 5 December 2013)

\begin{abstract}
Boron Neutron Capture Therapy (BNCT) is known to be a promising and new cancer therapy which can kill tumor cells suppressing damage to normal tissues. Recently, accelerator based neutron sources (ABNS) for BNCT are under development especially in Japan. For characterization of the neutron field, we carried out the series study concerning thermal/epi-thermal neutron spectrometry especially for ABNS based BNCT. In the present study, we tested a commercially available position sensitive ${ }^{3} \mathrm{He}$ proportional counter as the neutron detector combined with a measuring system using a multi-parameter MCA and a thermal/epi-thermal column designed and constructed for test measurements. From the results, the characteristics of the spectrometer were obtained through the basic experiments. Also, spectrum measurements were carried out with the thermal/epi-thermal neutron field. Agreement between the calculation and measurement was acceptable for both cases, showing that measurement would be possible for neutron energy from $0.01 \mathrm{eV}$ to $10 \mathrm{keV}$. The spectrometer is hence expected to be applied to characterization measurement of the neutron field in the real BNCT scene in the future.
\end{abstract}

(c) 2014 The Japan Society of Plasma Science and Nuclear Fusion Research

Keywords: cancer, radiation therapy, boron neutron capture therapy (BNCT), position sensitive proportional counter, ${ }^{3} \mathrm{He}$ detector, neutron spectrometer, epi-thermal neutron

DOI: $10.1585 /$ pfr.9.4401107

\section{Introduction}

Cancer is recognized as one of the most troublesome diseases worldwide. Especially in Japan, in the trend of cause of death, cancer (malignant neoplasm) caught up with cerebrovascular disease and became the first place after 1980 [1]. In Japan, the whole nation is tackling it very energetically. At present, there are several therapies for cancers known to be effective so far. The mainly utilized ones include operation, chemotherapy and radiation therapy. Since advantages and disadvantages exist for each therapy, a suitable one is chosen taking into account an individual case. Radiation therapy is known to be generally less invasive treatment and to have few side effects. On the other hand, radiation exposure cannot be avoided and is recognized as a serious defect. As the radiation therapy there are two methods utilized at present. One is a conventional therapy which uses X-ray or gamma-ray, named gamma-knife and cyber-knife, respectively. The other is ion beam therapy, which is quite new in Japan and is beginning to be spread recently. The former uses electrons as charged particle emitted via interaction of electromagnetic waves with matter (human body tissues) in order to kill a tumor. Because normal tissues and tumor cells are equally damaged by radiation, the exposure dose for the normal tissues should be suppressed as much as possible by concen-

author'se-mail: murata@eei.eng.osaka-u.ac.jp

*) This article is based on the presentation at the Conference on Laser and Accelerator Neutron Source and Applications (LANSA '13). trating the incident radiation beam within the tumor. The latter therapy utilizes a Bragg peak formed by charged particles. Energies of the charged particles are transferred intensively at around the Bragg peak. By changing the particle energy taking into account the tumor depth, the energy release can be controlled and concentrated at the tumor. As a result the damage of the normal tissues can be suppressed, killing the tumor cells at the same time.

In these circumstances, a new radiation therapy is being developed, i.e., boron neutron capture therapy (BNCT). Its possibility was pointed out just after discovery of neutron by Chadwick. Thereafter a lot of basic researches were carried out in nuclear reactor facilities. Clinical tests were performed with thermal neutrons of nuclear reactors for years. Recently, by using epi-thermal neutrons it became no need to conduct craniotomy. Since then, BNCT was really examined and refined as a low invasive cancer therapy. The number of clinical test cases drastically increased as a result. However, there exists a very severe problem especially in Japan that it is strictly not possible to construct a nuclear reactor in or close to a hospital. As well known, BNCT needs a strong neutron source and relies only on available experimental nuclear reactors. For this problem, not only in Japan but also in the world accelerator based neutron source (ABNS) is under investigation, because ABNS can be implemented in hospitals specifically in Japan.

At present, Kyoto University is leading in this field 
and carrying out clinical tests with a cyclotron based neutron source. There are several other projects running in Japan. Japan is now realizing practical treatment of BNCT with help of ABNS being as a pioneer of the world for eradication of cancers.

\subsection{Boron Neutron Capture Therapy (BNCT)}

Principle of BNCT is shown in Fig. 1. By administering specific medicines (BPA and/or BSH) including ${ }^{10} \mathrm{~B}$ in a human body, ${ }^{10} \mathrm{~B}$ can be accumulated in a tumor. Thermal or epi-thermal neutrons are then irradiated from outside of the human body. Thermal neutrons thus induce the next nuclear reaction to emit charged-particles $\left({ }^{4} \mathrm{He}\right.$ and ${ }^{7} \mathrm{Li}$ nuclei):

$$
\begin{aligned}
{ }^{10} \mathrm{~B}+\mathrm{n} & \rightarrow{ }^{4} \mathrm{He}+{ }^{7} \mathrm{Li}+2.79 \mathrm{MeV}(6 \%) \\
& \rightarrow{ }^{4} \mathrm{He}+{ }^{7} \mathrm{Li}^{*}+2.31 \mathrm{MeV}(94 \%)
\end{aligned}
$$

The emitted particles have energies of around $1 \mathrm{MeV}$, meaning the range in the human body is about $10 \mu \mathrm{m}$ which is similar to the size of cells. Hence, by concentrating ${ }^{10} \mathrm{~B}$ only in the tumor cells, only the tumor cells can be killed selectively. Exposure dose by neutrons are not neglected. However, since the reaction cross section of ${ }^{10} \mathrm{~B}(\mathrm{n}, \alpha)$ is exceptionally higher than common nuclides, the affection to the normal tissues can be suppressed substantially. Accumulation of ${ }^{10} \mathrm{~B}$ in the normal tissues is naturally not avoided. However, so-called T/B ratio (tumor to blood ratio of accumulated boron concentration) can be kept around 2 to 7 [2], meaning the dose to normal tissues is also kept very low. For these reasons, there exists a clear advantage in BNCT, that is, several-time irradiations are possible dislike other radiation therapies. In other words, the exposure dose to normal tissues of BNCT is very much suppressed compared to other radiation therapies. Although there are some other problems, e.g., supply of medicine containing boron, neutron source, etc., BNCT is recognized to be an attractive cancer therapy in the near future.

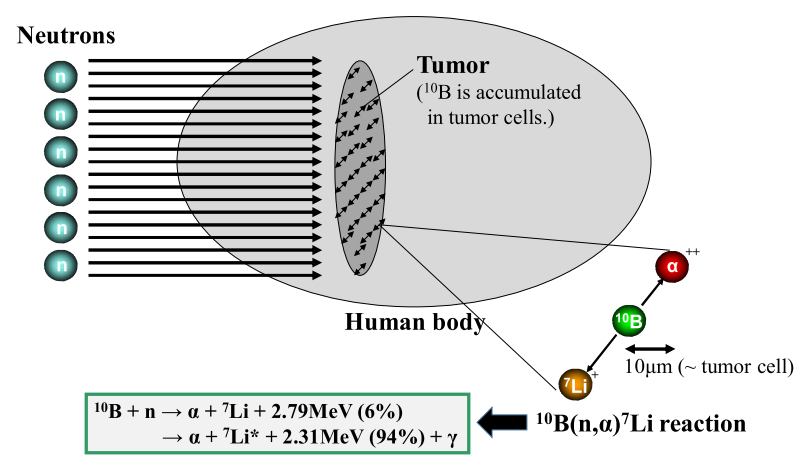

Fig. 1 Principle of BNCT.

\subsection{Objectives of the study}

At present an individual doctor performs BNCT with his/her own protocol in available nuclear reactors as a clinical study. It means there is no authorized standard protocol. It is strongly depending on the doctor who performs BNCT. Currently, the BNCT effect is estimated by making a product of the ${ }^{10} \mathrm{~B}$ concentration and the neutron flux intensity. The distribution of ${ }^{10} \mathrm{~B}$ concentration can be estimated by using positron emission tomography (PET) just before an actual BNCT [3]. On the other hand, the thermal neutron flux intensity can normally be estimated by simulation with a calculation code system like JCDS [4]. BNCT irradiation plan can be prepared in this way. However, in reality, after starting irradiation, with help of supplemented means like activation foils, small neutron detectors and so on, the ending time of irradiation is finally determined. Recently, it was pointed out that by this procedure the convalescence did not follow the expectation predicted before BNCT [2]. Practically, there were different convalescences observed at two Japanese available BNCT facilities of Kyoto University Research Reactor (KUR) and Japan Research Reactor 4 (JRR-4) of Japan Atomic Energy Agency even with an equal protocol of BNCT. This fact indicates that the current BNCT irradiation planning can give just a reference plan for BNCT. The key issue is that it is not possible to know the real effect during irradiation in real time. For this problem, several studies were carried out so far with semiconductor detectors or scintillators [5-10]. However, at the same time it is quite crucial to improve the accuracy of the BNCT irradiation plan. Key values are boron concentration and neutron flux distribution used to estimate the BNCT effect. As described just before, the former information can be obtained experimentally by PET. However, the latter is estimated just by a simulation. For the simulation calculation, the source term is indispensable as an initial condition. It means accurate neutron field characterization is indispensable beforehand in addition to the boron concentration measurement. In nuclear reactors in which BNCT is carried out, i.e., KUR or JRR-4, the thermal/epi-thermal neutron field is fairly well characterized. However, if ABNS for BNCT will be realized, the neutron field can vary depending on accelerator, moderator, target, incident particle and its energy. The energy spectrum in the irradiation room has to be accurately characterized ideally within several $\%$.

In the present paper, following the introduction of BNCT for readers of Plasma and Fusion Research in Chap. 1, is described in the following chapters the present status of development of a low energy neutron spectrometer which covers energies of thermal to epi-thermal region commonly used for BNCT.

\section{Theory [11]}

As well known, no straightforward way exists to measure neutron spectrum. Especially low-energy neutron 


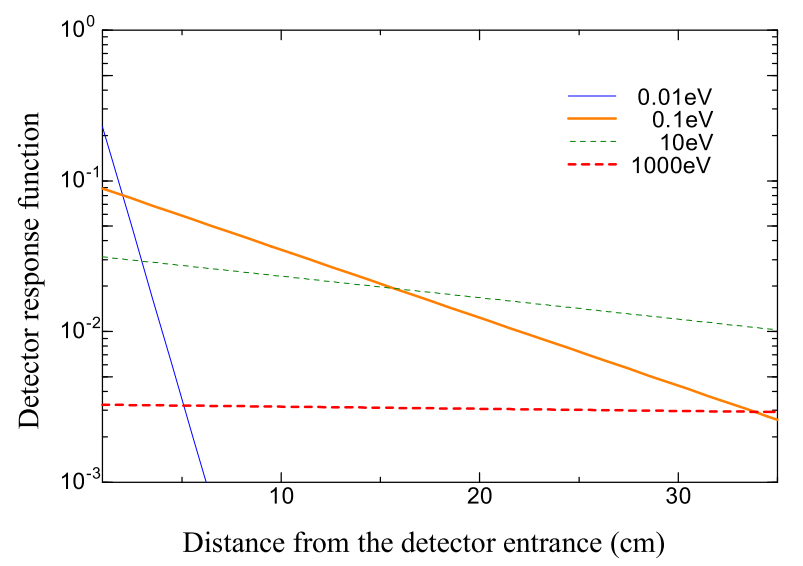

Fig. 2 Detector response for several neutron energies.

spectrum measurement is critically difficult, because the energy of neutrons, being around $\mathrm{eV}$ or $\mathrm{keV}$, is so smaller than $\mathrm{Q}$ values of nuclear reactions normally used to detect low-energy neutrons. Commonly neutron detectors like proportional counters aiming at detection of low-energy neutrons are used to just count the number of incident neutrons, not the energy spectrum.

The important point is to find physical quantity which can expand small energy difference in the low-energy region. A possible physical quantity is reaction cross section, because some nuclear reactions have very large reaction cross section values in the low-energy region and the values decrease drastically with increase of energy. In addition, there exists clear one-to-one correspondence between energy and the cross section value in such reactions, because they have an inverse-velocity cross section curve in the low-energy region.

The reaction cross section difference may create a detection position (depth) difference especially in a gas counter, because lower energy neutrons can be captured immediately after entering into the detector, however, higher energy neutrons can transmit into deeper places until captured. With a position sensitive gas proportional counter, we can measure reaction depth distribution, which possesses the neutron energy information. The neutron spectrum could be reproduced from the measured reaction depth distribution with a suitable response function, that is, the reaction depth distribution for each neutron energy bin as shown in Fig. 2.

\section{Developed Spectrometer}

Requirements for the presently utilized position sensitive proportional counter include (1) higher detection gas pressure, (2) longer detector tube and (3) larger diameter, in order to improve its detection efficiency and energy resolution. At first, we had selected ${ }^{3} \mathrm{He}$ gas and a prototype ${ }^{3} \mathrm{He}$ detector produced by ourselves so as to meet these requirements [12]. However, due to so-called "he-

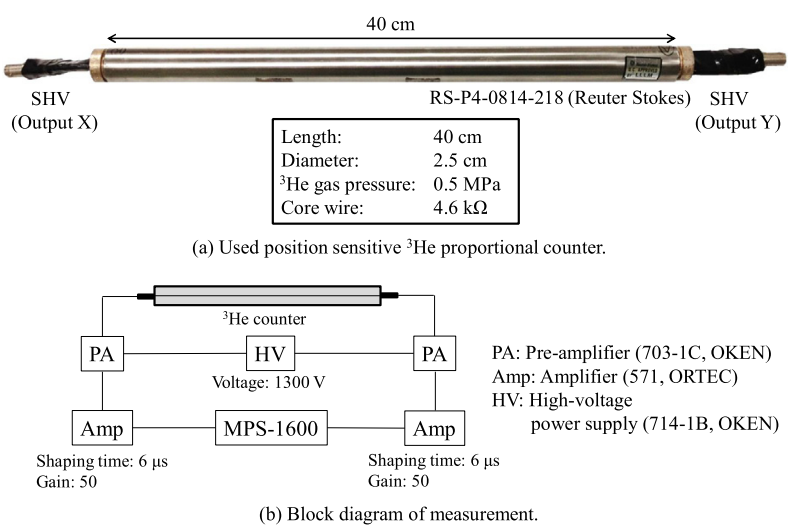

Fig. 3 Presently used counter and measuring system.

lium crisis", it became extremely difficult to obtain ${ }^{3} \mathrm{He}$ gas. We then decided to use a commercial-base position sensitive proportional counter shown in Fig. 3 (a) instead to complete the present spectrometer system. The length and diameter of the detector are $40 \mathrm{~cm}$ and $2.5 \mathrm{~cm}$, respectively. The ${ }^{3} \mathrm{He}$ gas pressure is $0.5 \mathrm{MPa}$. As detailed later, to measure the detection position (depth) distribution of an incident low-energy neutron, the detector is so set that incident neutrons enter into the detector from one of two detector ends, i.e., the neutron beam direction is parallel to the detector axis. As shown in Fig. 3 (b), two signals from both ends of the detector are measured by two pairs of preamplifier and main-amplifier and fed to a multi-parameter system (MPS-1600 (Laboratory Equipment Corporation)) to obtain a two-dimensional contour which has energy information.

\section{Experimental}

As preparation, at first we designed and developed thermal/epi-thermal neutron columns to supply appropriate neutron fields for examining the basic feature of the present ${ }^{3} \mathrm{He}$ detector. This experiment is called "basic measurement". Then we also designed and developed a detector shield to remove background neutrons coming from the side surface of the detector. Experiments with the completed spectrometer consisting of the ${ }^{3} \mathrm{He}$ detector and the developed shield are called "spectrum measurement" in this paper.

\subsection{Thermal/epi-thermal columns}

An AmBe (46.3 GBq) was used as a neutron source for constructing thermal/epi-thermal neutron columns. From the experimental result of the authors' group, the neutron intensity was found to be $2.4 \times 10^{6} \mathrm{n} / \mathrm{sec}$ [13]. The whole design was conducted with a general purpose Monte Carlo code, MCNP5.

As the thermal column, we utilized a graphite column $\left(100 \times 100 \times 100 \mathrm{~cm}^{3}\right)$ at the OKTAVIAN facility of Osaka University, Japan. From the parameter survey calculations 


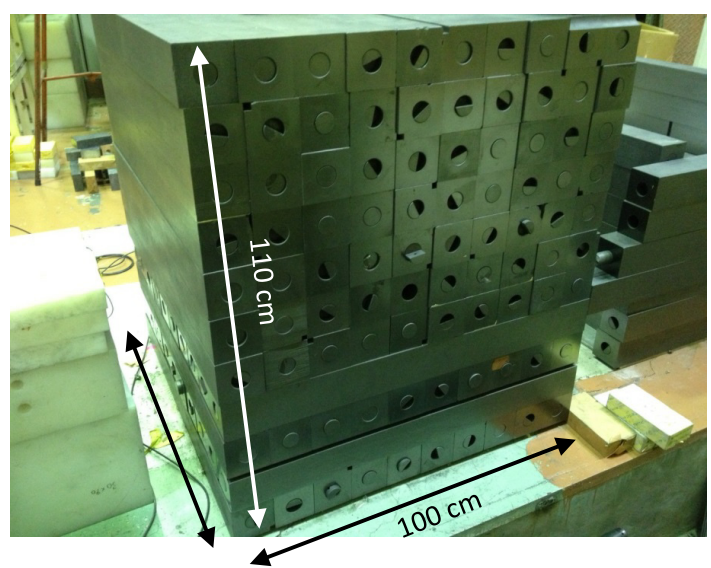

Fig. 4 Produced thermal neutron column.

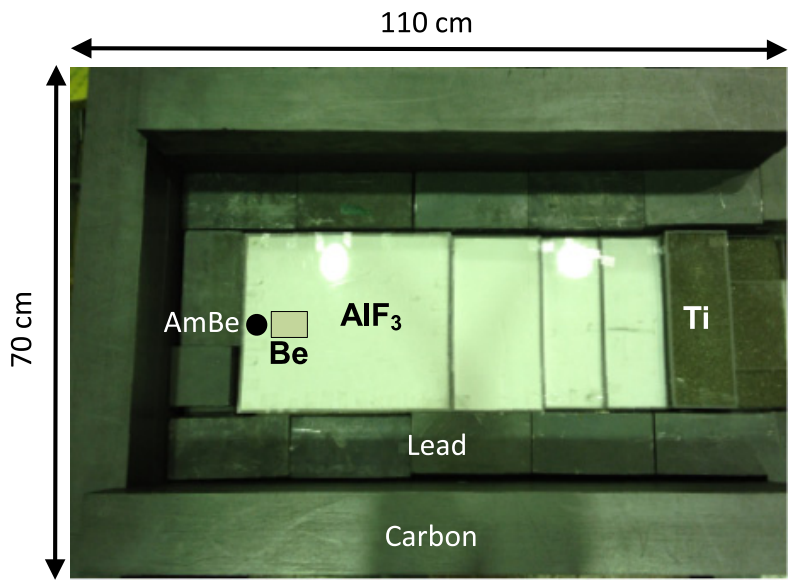

Fig. 5 Produced epi-thermal neutron column. The AmBe source and beryllium are set inside the case of $\mathrm{AlF}_{3}$ powder.

the neutron source was placed at the depth of $40 \mathrm{~cm}$ from the column surface [14]. As for the epi-thermal column we changed moderator material inside the graphite column from the thermal column. In the column aluminum fluoride was used as a main moderator. However, because high energy part was not negligible, titanium layer was added at the exit of the aluminum fluoride to remove neutrons over $10 \mathrm{keV}$ [15]. Lead and graphite layers surrounding the whole moderator were arranged as an effective reflector. Photos of the thermal and epi-thermal columns are shown in Figs. 4 and 5.

\subsection{Spectrometer arrangement}

For the basic measurement, the ${ }^{3} \mathrm{He}$ detector was so set up that the neutron beam direction became perpendicular to the detector axis, (1) to confirm whether the detection position difference would appear in the measured two-dimensional spectrum and (2) to assign the real detection position to the channel of the measured detection position distribution by using an appropriate neutron-absorber (cadmium) collimator.

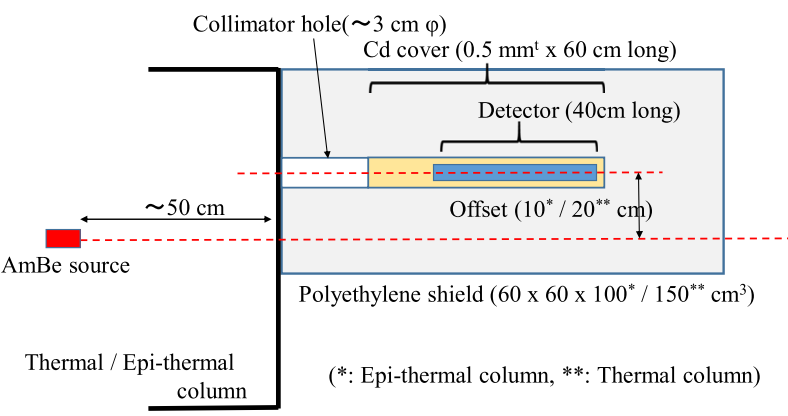

Fig. 6 Arrangement of the spectrometer assembly.

The spectrum measurement was carried out with an arrangement shown in Fig. 6. In the arrangement, there are two points. One is that neutrons to be measured should enter only from one of both ends parallel to the detector axis. Thus, the side of the detector should thoroughly be shielded. Practically, it was covered with a cadmium sheet inside and a polyethylene layer was set outside the cadmium. This side shield set is longer than the detector itself so that it plays also a role of collimator to collimate neutrons coming into the spectrometer. The other point is that the detector axis should not view the AmBe neutron source directly through the collimator hole, otherwise direct high energy neutrons enter through the hole from the source and cause a lot of noises. We thus arranged the detector having an offset as shown in Fig. 6. However, even with this spectrometer assembly, background neutrons are not removed perfectly since the source intensity is not so strong. Cadmium cup and polyethylene plug for thermal and epi-thermal neutron measurements, respectively, are thus put in front of the collimator entrance in order to extract the spectrum by making the difference between measured results with and without the cup or plug.

\section{Results and Discussion}

\subsection{Two-dimensional spectrum}

An example of the measured two-dimensional spectrum is shown in Fig. 7. The horizontal axis shows one of the two signals and the vertical axis shows the other. In this measurement, no cadmium collimators as neutron absorber and no neutron shields were used, meaning that neutrons are entering the detector from the side surface uniformly and perpendicularly. It was confirmed from the figure that the detection position information could successfully be extracted.

\subsection{Detection position identification}

To confirm one-to-one correspondence between the position (coordinates) in the measured two-dimensional spectrum and the real detection position in the detector, the detector was covered with a cadmium collimator to shield thermal neutrons. Neutrons only entering from open windows of $1 \mathrm{~cm}$ width of the collimator can be detected. The 


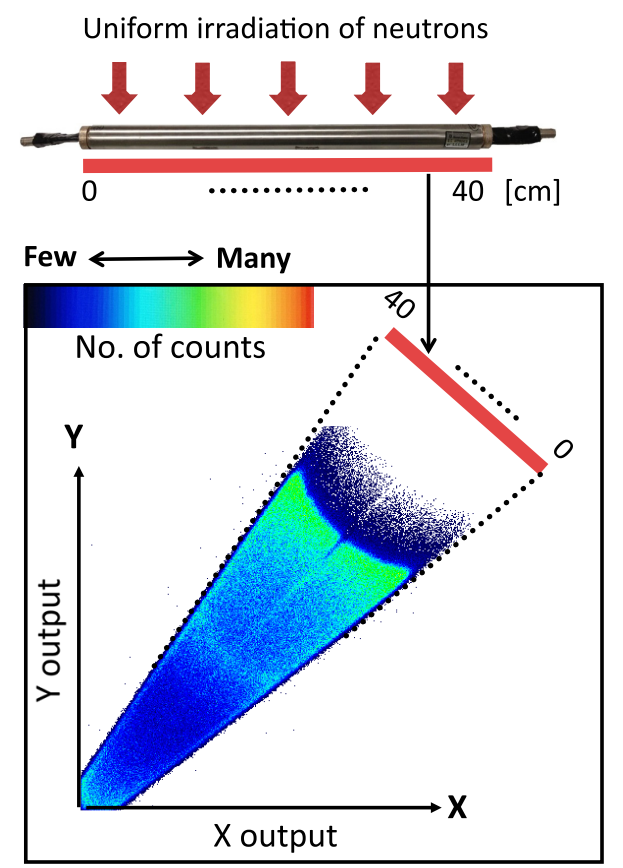

Fig. 7 Two-dimensional contour of reaction depth distribution (Uniform incidence from the side surface of the detector).

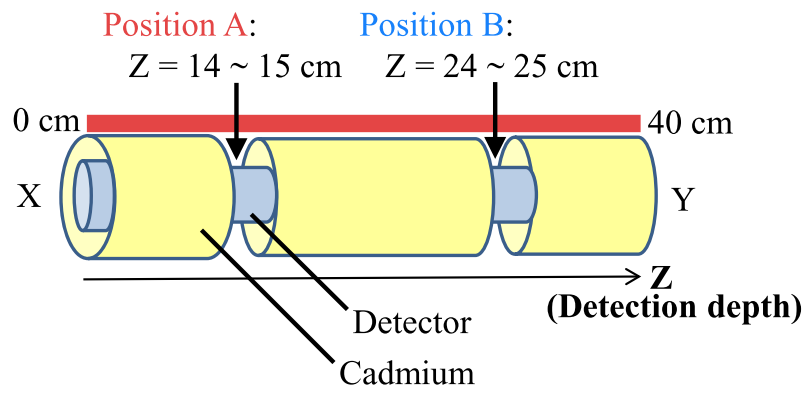

Fig. 8 Schematic view of the detector set-up for detection position identification.

schematic view of the detector set-up is shown in Fig. 8. In this case, center positions of two open windows (A and $\mathrm{B}$ in the figure) were $14.5 \mathrm{~cm}, 24.5 \mathrm{~cm}$ from one side edge (X), respectively, at which the corresponding spectrum was observed as in Fig. 9. We then projected the counts of the two-dimensional spectrum along a line starting from the origin as a function of the angle from $\mathrm{x}$-axis. The result is shown in Fig. 10, in which two clear peaks are observed corresponding to the positions where no cadmium covers exist. By repeating this experiment, we successfully assigned the real detection position from $0 \mathrm{~cm}$ to $40 \mathrm{~cm}$ in the detector to the angle in Fig. 9.

\subsection{Spectrum measurement}

Figure 11 shows a measured neutron energy spectrum which was obtained by unfolding a detection depth distribution measured with the thermal column in the setup

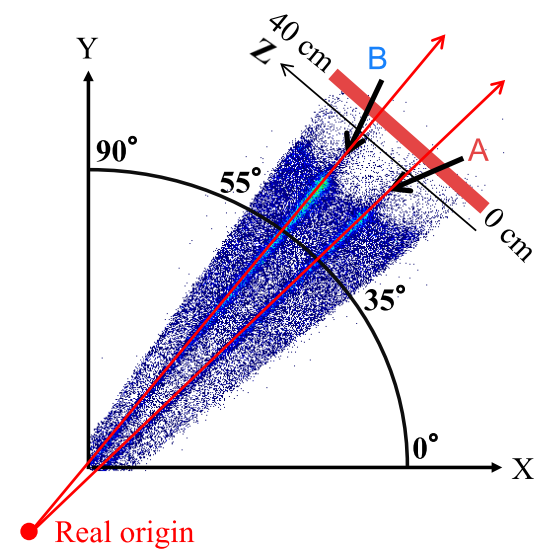

Fig. 9 Two-dimensional contour measured in the arrangement in Fig. 8.

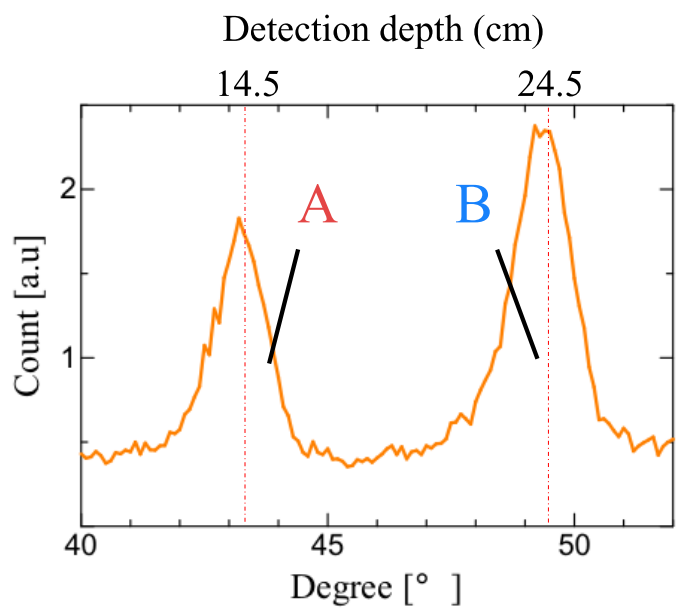

Fig. 10 Projection of plot in Fig. 9 along a line starting from the origin as a function of the angle from $\mathrm{x}$-axis.

shown in Fig. 6. Also, the arrangement of Fig. 6 was numerically analyzed with MCNP5 to estimate the spectrum to be obtained in the measurement. The calculated result is shown together with the measured result in Fig. 11. Agreement of both spectra is fairly well, indicating spectrum measurement around thermal neutron energy range is possible with this spectrometer. As a result, the lower measurable limit is confirmed to be about $0.01 \mathrm{eV}$. Slight discrepancy seen in the figure is expected to be caused mainly by a statistical uncertainty, because the used $\mathrm{AmBe}$ is not so intense and it took several days to complete one spectrum measurement. The statistical error is estimated to be $10 \sim 40 \%$ though not specified explicitly in the figure.

Figure 12 shows the result for the case of epi-thermal neutron measurement. Fairly good agreement is obtained between measurement and calculation. Although fine structure is not reproduced in the spectrum similar to the thermal case due to statistical accuracy problem, measurement up to around $10 \mathrm{keV}$ was confirmed to become possible. It took also several days to carry out the complete 


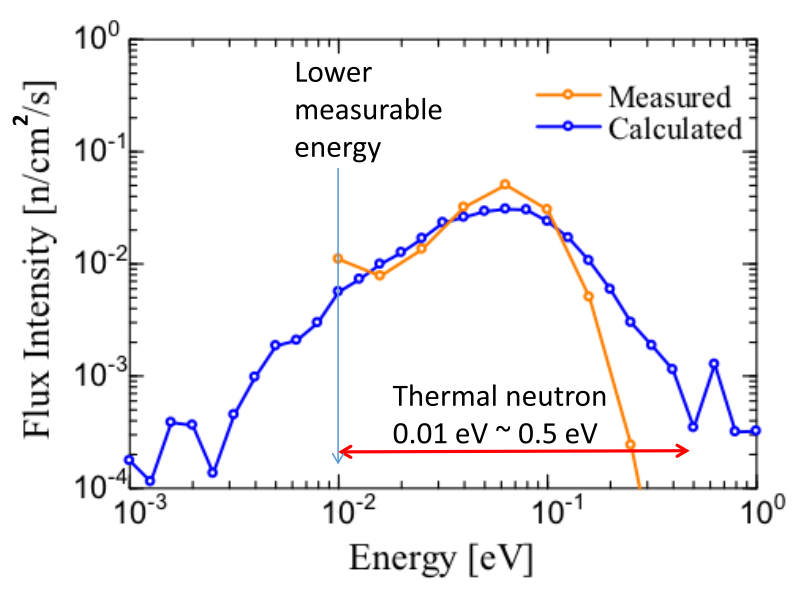

Fig. 11 Measured thermal neutron spectrum compared with the calculation result.

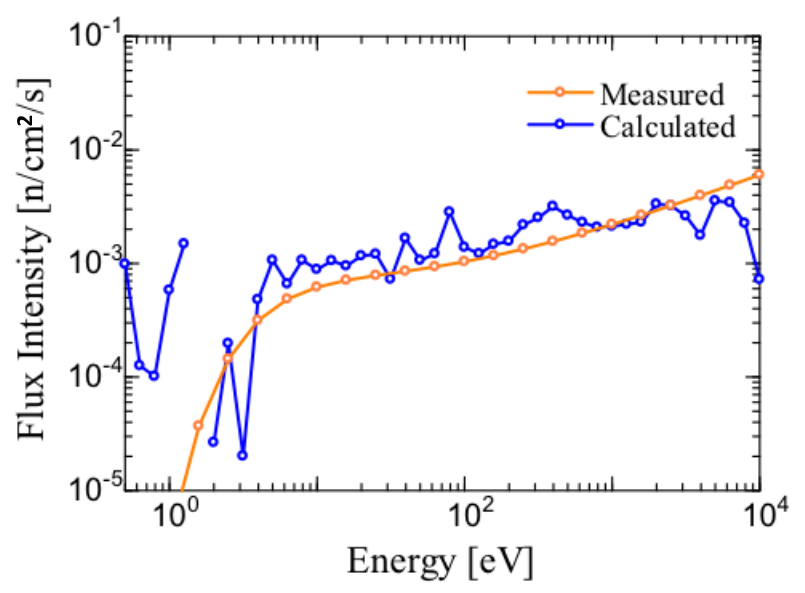

Fig. 12 Measured epi-thermal neutron spectrum compared with the calculation result.

measurement in the epi-thermal case. It is thus required to conduct more accurate experiments using a stronger neutron source. From the series experimental studies so far, it was confirmed that spectrum measurement covering thermal to epi-thermal energies with the present spectrometer was possible in principle though the accuracy is still low compared to the goal of several \%. The spectrometer is expected to be applied to characterization measurement of the neutron field in the real BNCT scene in the future.

\section{Conclusion}

We carried out the series study concerning a thermal/epi-thermal neutron spectrometer especially for BNCT. In the present study, we tested a commercially available position sensitive ${ }^{3} \mathrm{He}$ proportional counter as the neutron detector combined with a measuring system with a multi-parameter MCA and a thermal/epi-thermal column designed and constructed for test measurements.

From the results of the basic measurements, the detection position information could successfully be obtained as a two dimensional spectrum. And, the real detection position could be assigned to the position in the measured detection position. We also carried out spectrum measurements with thermal/epi-thermal neutron fields by the present spectrometer so arranged that incident neutrons entered parallel to the detector axis. The agreement between calculation and experiment was acceptable for both cases, showing that the dynamic range of the detector was $0.01 \mathrm{eV}$ to $10 \mathrm{keV}$. However, further experiments are required to confirm it more accurately with stronger neutron sources. The spectrometer is expected to be applied to characterization measurement of the neutron field in the real BNCT scene in the future.

[1] Ministry of Health, Labor and Welfare, Japan, http://www. mhlw.go.jp/toukei/saikin/hw/jinkou/geppo/nengai11/ kekka03.html\#k3_2 (in Japanese).

[2] I. Kato, private communication, Osaka University (2008).

[3] Y. Imahori et al., J. Nucl. Med. 39, 325 (1998).

[4] H. Kumada et al., J. Physics: Conf. Series 74, 021010 (2007).

[5] W. Verbakel et al., Nucl. Instrum. Methods Phys. Res. Sect. A 394 (1-2), 163 (1997).

[6] T. Kobayashi et al., Med. Phys. 27(9), 2124 (2000).

[7] P. Munck af Rosenschöld et al., J. Instrum. 1, 1 (2006).

[8] D.M. Minsky et al., Appl. Radiat. Isot. 67(7-8), S179 (2009).

[9] I. Murata et al., Appl. Radiat. Isot. 69, 1706 (2011).

[10] I. Murata et al., Progress in Nucl. Sci. Technol. 1, 267 (2011).

[11] I. Murata and H. Miyamaru, Nucl. Instrum. Methods Phys. Res. A589, 445 (2008).

[12] M. Ito and I. Murata, "Thermal/epi-thermal neutron spectrometer with a ${ }^{3} \mathrm{He}$ position sensitive proportional counter," Proc. 2010 Annual Symposium on Nucl. Data, Nov. 25-26, 2010, C-CUBE, Chikushi Campus, Kyushu University, JAEA-Conf 2011-002, pp.95-100 (2011).

[13] I. Murata et al., "Neutron and Gamma-ray Source-Term Characterization of AmBe Sources in Osaka University," to be published in Progress in Nucl. Sci. Technol. (2013).

[14] K. Harada and I. Murata, "Design and Construction of A Thermal Column Using An Am-Be Neutron Source for BNCT studies," Proc. of the 2011 Annual Symposium on Nucl. Data, Nov. 16-17, 2011, RICOTTI, Tokaimura, Ibaraki-ken, Japan, JAEA-Conf 2012-001, pp.129134 (2012).

[15] K. Harada, Master thesis, Osaka University (2011) (in Japanese). 